\title{
Variação espacial entre verão e inverno da meiofauna do Canal de São Sebastião - SP
}

\author{
(Spatial variation between summer and winter of the meiofauna from \\ São Sebastião Channel (SP))
}

\author{
Adriana M. Moellmann*; Thaïs N. Corbisier \& Rita R. Curvelo \\ Instituto Oceanográfico da Universidade de São Paulo \\ (Caixa Posta 66149, 05315-970 São Paulo, SP, Brasil) \\ $\left(^{*}\right)$ e-mail:adrimoellmann@ig.com.br
}

- Abstract: Spatial and seasonal variation of the density and biomass of meiofauna at higher taxonomic group levels were studied and related to sediment characteristics and water masses in São Sebastião Channel. The sampling was done at ten stations in the channel in February (summer) and August (winter) 1994. In the summer, the mean density was $605 \pm 442$ individuals per $4.9 \mathrm{~cm}^{2}$, and in the winter, the mean density decreased to $213 \pm 181$ individuals per $4.9 \mathrm{~cm}^{2}$. Total mean biomass ranged from $184.7 \pm 156.8 \mu \mathrm{g} \mathrm{x} 4.9 \mathrm{~cm}^{-2}$ to $66.6 \pm 48.9 \mu \mathrm{g} \mathrm{x} 4.9 \mathrm{~cm}^{-2}$, showing similar pattern as the density. Nematoda was the main group at most stations followed by Copepoda, as function of sediment type. Meiofauna characteristics at São Sebastião Channel reflected both the heterogeneous spatial distribution of the sediment along the channel and its seasonal variation, as well.

- Resumo: A variação espacial e temporal da densidade e biomassa da meiofauna, em nível de grupos taxonômicos superiores, foi analisada e relacionada com as características do sedimento e as massas de água no Canal de São Sebastião. As coletas foram realizadas em dez estações, em fevereiro (verão) e agosto (inverno) de 1994. No verão, a densidade média foi de $605 \pm 442$ ind $x 4,9 \mathrm{~cm}^{-2}$ e no inverno, $213 \pm 181$ ind $\times 4,9 \mathrm{~cm}^{-2}$. A biomassa média total variou de $184,7 \pm 156,8 \mu \mathrm{g} \times 4,9$ $\mathrm{cm}^{-2}$, no verão, diminuindo para $66,6 \pm 48,9 \mu \mathrm{g} \mathrm{x} 4,9 \mathrm{~cm}^{-2}$, no inverno, mostrando padrão semelhante ao da densidade. Nematoda foi o grupo dominante em quase todas as estações, seguido de copepoda, em função do sedimento predominante. As características gerais da meiofauna na Canal de São Sebastiấo refletiram tanto a heterogeneidade espacial da distribuição dos sedimentos ao longo do canal, como também a sua variação temporal.

- Descriptors: Meiofauna, Ecology, Continental shelf, Sediment, São Sebastião Channel, Brazil.

- Descritores: Meiofauna, Ecologia, Plataforma continental, Sedimento, Canal de São Sebastião, Brasil.

\section{Introdução}

Em sistemas de plataforma continental, a composição taxonômica, a densidade e a biomassa da meiofauna são reflexos de fatores ambientais tais

$\overline{\text { Contr. no. } 842}$ do Inst. oceanogr. da Usp. como a profundidade (Tenore et al., 1978; Alongi, 1989), granulometria do sedimento (Hanson et al., 1981), entrada de nutrientes e/ou detritos provenientes de sistemas frontais ou de origem continental (Alongi, 1989, 1990), contribuição de sedimentação pelágica (Rudnick et al., 1985) e ocorrência de distúrbios físicos no sedimento em 
função do hidrodinamismo (Aller \& Aller, 1986; Guidi-Guilvard \& Buscail, 1995; Thistle et al., 1995), entre outros. Em especial, a passagem de tempestades pode causar a remobilização dos sedimentos, determinando a remoção de grande parte dos pequenos metazoários bentônicos, em particular a meiofauna (Thistle et al., op. cit.), refletindo-se na estrutura destas comunidades.

No ecossistema de plataforma da região sudeste brasileira, há grande variação sedimentar em função da fisiografia e do hidrodinamismo (PiresVanin, 1992 e 1993; Mahiques et al., 1999), ocorre a entrada de sistemas frontais, que enriquecem o sistema na primavera-verão (Castro Filho et al., 1987; Pires-Vanin, 1993), e também a passagem de frentes frias, associadas a ventos de sul-sudoeste no inverno, que remobilizam os sedimentos junto ao fundo na plataforma interna (Tessler, 1988). Todos estes aspectos tem efeito sobre as comunidades bentônicas (Pires-Vanin, 1993; Paiva, 1993; Corbisier, 1993).

No canal está instalado um dos maiores terminais petrolíferos do Brasil, o DTCS (Duto e Terminais Centro Sul) da Petrobrás, e um porto comercial. Além disso, há o despejo do emissário de esgoto municipal da região. Todos estes fatores, mais o interesse turístico, tornam a região uma área de intensa influência antrópica.
O objetivo deste estudo foi analisar a variação espaço-temporal da densidade e biomassa da meiofauna e de sua composição taxonômica, em nível de grupos superiores, no Canal de São Sebastião, e relacionar estas características a fatores ambientais, como as características do sedimento e de massas d'água.

\section{Material e métodos}

O Canal de São Sebastião situa-se entre as longitudes $45^{\circ} 19^{\prime} \mathrm{W}$ e $45^{\circ} 30^{\prime} \mathrm{W}$, e as latitudes de $23^{\circ} 41^{\prime}$ 'S e $23^{\circ} 53.5^{\prime}$ S (Fig. 1) e separa a Ilha de São Sebastião do continente adjacente, possuindo um comprimento aproximado de $24 \mathrm{~km}$ e estendendo-se na direção SW-NE. Ele apresenta larguras de $5,8 \mathrm{~km}$ na extremidade norte e $6,4 \mathrm{~km}$ na extremidade sul, e na região central estreita-se para $2,0 \mathrm{~km}$. A profundidade máxima é de aproximadamente $45 \mathrm{~m}$. O eixo do canal é deslocado para o lado da Iha de São Sebastião (Furtado, 1995).

Foram amostradas 10 estações em março (verão) e agosto (inverno) de 1994, com o B/Pq "Véliger II". Em cada estação, o sedimento foi coletado com pegador tipo van Veen $\left(0,10 \mathrm{~m}^{2}\right) \mathrm{e}$

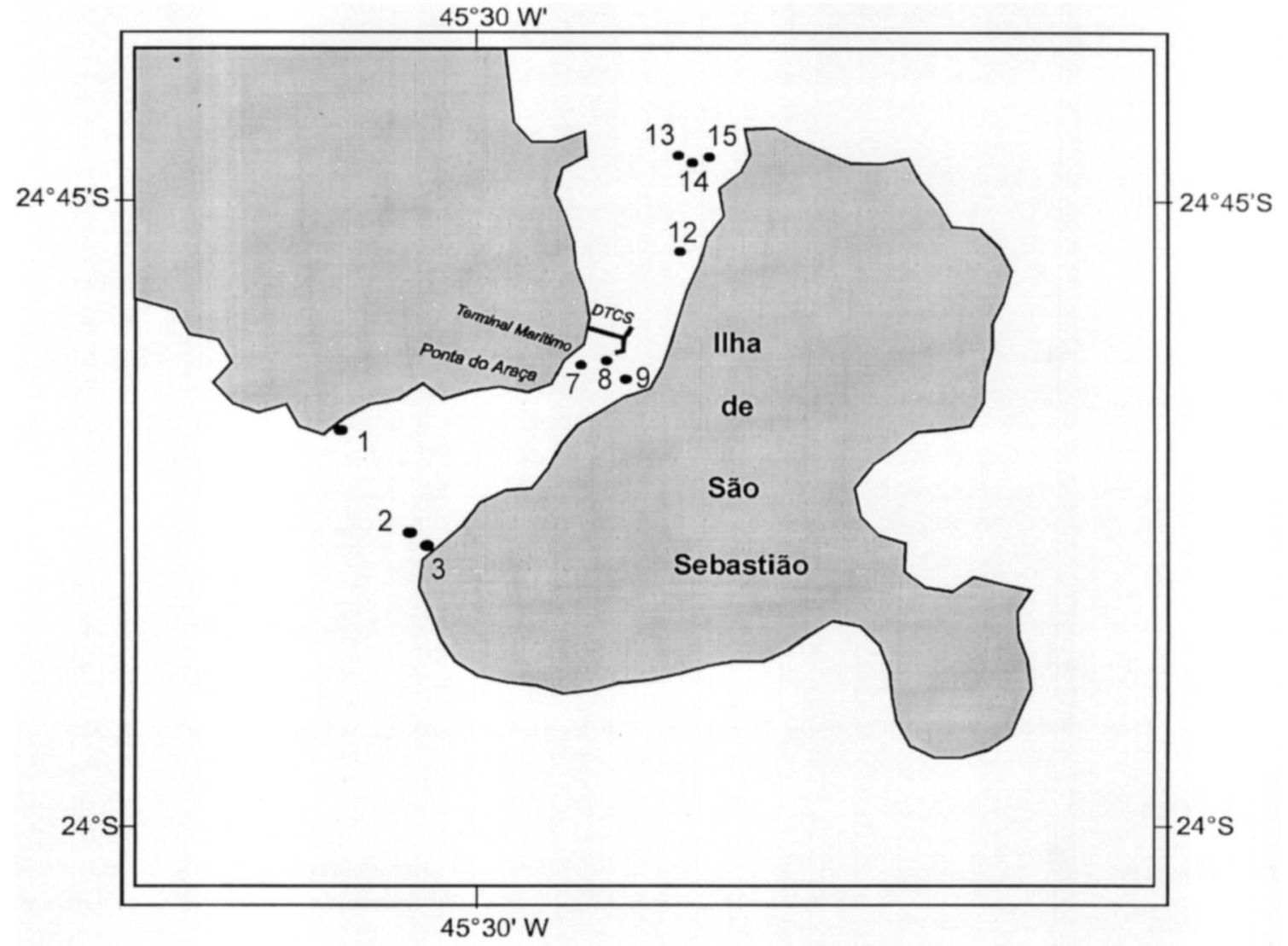

Fig. 1. Área de estudo e localização das estações de coleta. 
foram retiradas 4 réplicas com tubos de PVC de 2,5 $\mathrm{cm}$ de diâmetro $\left(4,9 \mathrm{~cm}^{2}\right.$ de área $)$ e $10 \mathrm{~cm}$ de altura. $\mathrm{A}$ bordo as réplicas foram fixadas com formol $5 \%$ neutralizado. Foi retirada também de cada estação uma amostra de sedimento para a realização de análise sedimentológica.

Em laboratório, o material de cada amostra foi lavado e peneirado através de uma série de quatro peneiras de telas de tamanhos diferentes $(500,250$, 125 e $62 \mu \mathrm{m})$ a fim de separar a fauna em diferentes categorias de tamanho (Pfannkuche \& Thiel, 1988). O material retido em cada peneira foi novamente fixado, corado com Rosa de Bengala e triado sob estereomicroscópio. Os grupos foram identificados até níveis taxonômicos superiores e contados. O nível taxonômico superior da meiofauna foi utilizado em função de refletir as principais condições ambientais (Bartlett et al., 1987; Coull \& Chandler, 1992).

A análise de sedimento foi feita segundo a metodologia descrita em Suguio (1973) após a retirada dos carbonatos. $\mathrm{O}$ teor de carbono orgânico foi determinado por oxidação com $\mathrm{K}_{2} \mathrm{CrO}_{7}$ e titulação do dicromato restante com ácido clorídrico (Gaudette et al., 1974). Os carbonatos foram determinados pelo método de dissolução ácida em toda a amostra. Em cada estação também foram determinadas a temperatura e a salinidade da água de fundo.

A biomassa foi estimada, em termos de peso seco livre de cinzas, para cada grupo taxonômico, separados em categorias de tamanho, a partir dos pesos médios individuais segundo dados de Widbom (1984), Rudnick et al. (1985) e Bodin et al. (1989).

As análises estatísticas para procurar diferenças significativas entre as densidades da meiofauna foram realizadas através do teste não paramétrico de Kruskal-Wallis. $\mathrm{O}$ teste a posteriori de comparações múltiplas não paramétrico tipo Tukey foi utilizado para procurar diferenças significativas (Zar, 1996). A Análise de Correspondência Canônica (ACC) foi usada para ordenar simultaneamente os principais grupos da meiofauna, as amostras das estações de coleta e as variáveis ambientais (Jongman et al., 1987), utilizando-se o programa CANOCO versão 3.10 (ter Braak, 1988, 1990). As densidades foram transformadas por $\log (x+1)$ e os dados ambientais, padronizados pela transformação $[(\mathrm{x}-$ $\left.\left.\mathrm{x}_{\mathrm{m}}\right) / \mathrm{dp} . \mathrm{x}\right]$.

\section{Resultados}

\section{Dados ambientais}

No verão, a área esteve sob influência da Água Costeira, exceto a estação mais profunda no centro do canal (estação 8 ) onde a baixa temperatura da água de fundo indicou resquícios da presença da Água Central do Atlântico Sul, durante a coleta (Tab. 1). O sedimento variou bastante, desde areia grossa na estação 14 e areia média nas estações 3,9 e 13, dispostas ao longo do lado insular, a areia argilosa

Tabela 1. Dados ambientais das estações do Canal de São Sebastião.

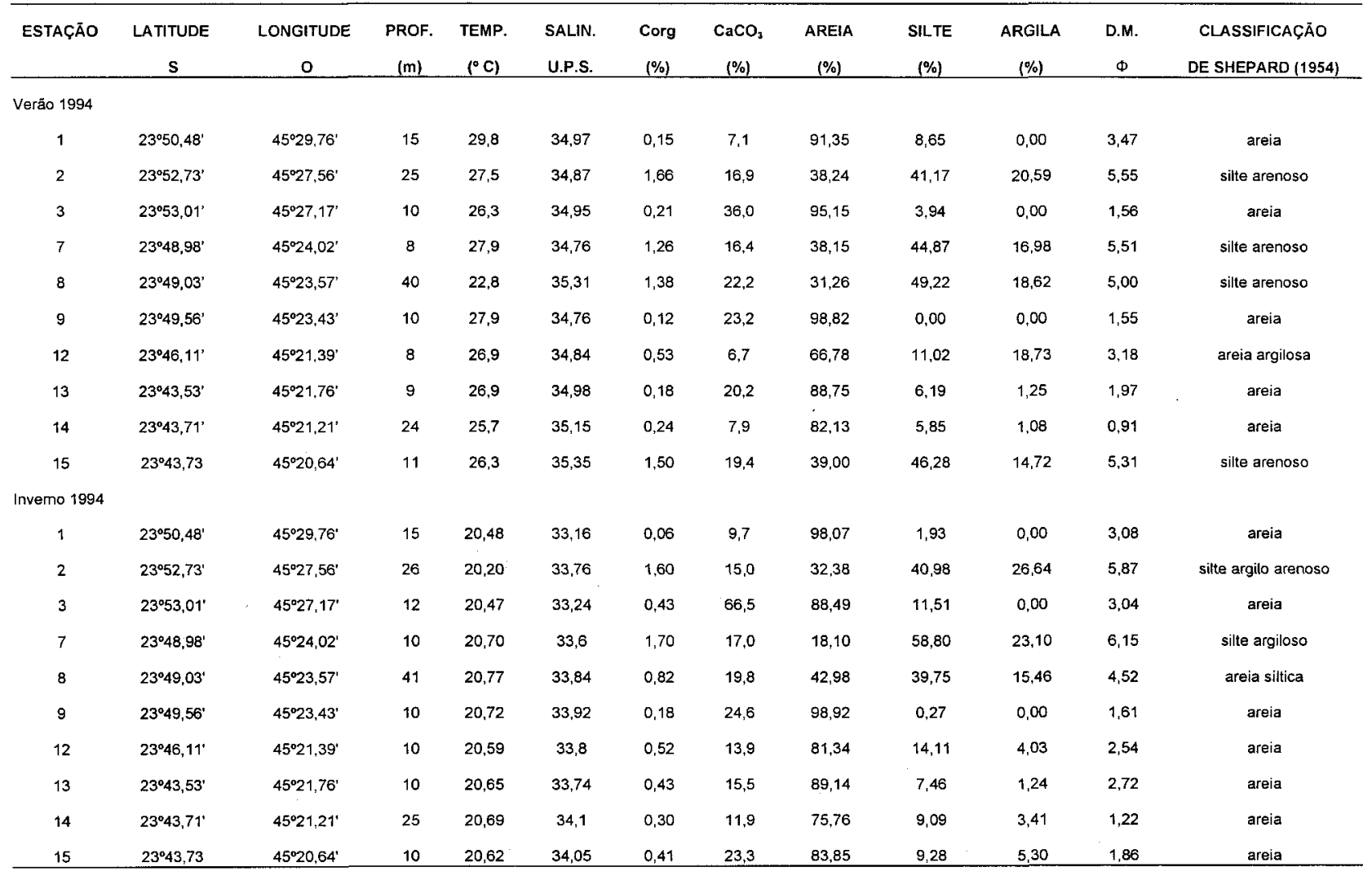


nas estações 1 e 12, e silte arenoso nas estações 2, 7,8 e 15 , ao longo do canal. Os valores de carbonatos biodetríticos variaram de $6,7 \%$, na estação 12 , a $36,0 \%$ na estação 3 . Os valores de carbono orgânico foram maiores nas estações $2,7,8$ e $15(>1,0 \%)$.

No inverno, os dados de temperatura e salinidade na água de fundo foram homogêneos na área e inferiores aos dados verão (Tab. 1). O sedimento variou de areia média nas estações $9,14 \mathrm{e}$ 15 , areia fina nas estações 12 e 13 , areia muito fina nas estações 1 e 3, areia síltica na estação 8 , silte argilo arenoso na estação 2 , e silte argiloso na estação 7. Os valores de carbonato foram mais altos somente na estação $3(66,5 \%)$, tendo variado de 9,7 a $24,6 \%$ no restante do canal. Os valores de carbono orgânico foram maiores nas estações 2 e 7 $(>1,6 \%)$.

Entre o verão e o inverno, observaram-se alterações nas características do sedimento na. maioria das estações, com exceção das estações 2 e 7 (Tab. 1).

\section{Meiofauna}

No verão, observaram-se 16 grupos taxonômicos no canal (Tab. 2). Os Nematoda constituíram $79,0 \%$ do total, enquanto que os Copepoda constituíram $11,4 \%$, larvas de Crustacea $3,9 \%$, Polychaeta $2,4 \%$ e Kinorhyncha $2,3 \%$. Cada um dos demais grupos correspondeu a menos de $1 \%$ do total da meiofauna. As estações que apresentaram maior diversidade de grupos foram as estações 3, 8, 9, 13 e 14, onde também houve maior representatividade dos Copepoda (Fig. 2).

Neste período, foi observada uma densidade média ( \pm desvio padrão) de $608 \pm 443$ indivíduos por $4,9 \mathrm{~cm}^{2}$, sendo destes $478 \pm 386$ indivíduos do grupo Nematoda (Tab. 2). A densidade média da meiofauna variou de $149 \pm 124$ a $1311 \pm 87$ indivíduos por $4,9 \mathrm{~cm}^{2}$. As estações com maior densidade de meiofauna foram as estações $8 \mathrm{e} 1 \mathrm{e}$ as densidades mais baixas foram observadas nas

Tabela 2. Média das densidades da meiofauna ( $\mathrm{n}^{-}$ind. $4,9 \mathrm{~cm}^{-2}$ ) nas estações de coleta do Canal de São Sebastião - Verão 1994 ( $\mathrm{X}=$ média; $\mathrm{DP}=$ desvio padrão).

\begin{tabular}{|c|c|c|c|c|c|c|c|c|c|c|c|c|c|c|c|c|c|c|c|c|c|c|}
\hline \multirow{2}{*}{$\frac{\text { ESTAÇÖES }}{\text { GRUPOS }}$} & \multicolumn{2}{|c|}{01} & \multicolumn{2}{|c|}{02} & \multicolumn{2}{|c|}{03} & \multicolumn{2}{|c|}{07} & \multicolumn{2}{|c|}{08} & \multicolumn{2}{|c|}{09} & \multicolumn{2}{|c|}{12} & \multicolumn{2}{|c|}{13} & \multicolumn{2}{|c|}{14} & \multicolumn{2}{|c|}{15} & \multicolumn{2}{|c|}{ TOTAL } \\
\hline & $x$ & $\pm \mathrm{DP}$ & $x$ & $\pm \mathrm{DP}$ & $x$ & $\pm D P$ & $x$ & $\pm \mathrm{DP}$ & $x$ & $\pm \mathrm{DP}$ & $x$ & $\pm \mathrm{DP}$ & $x$ & $\pm \mathrm{DP}$ & $x$ & $\pm \mathrm{DP}$ & $x$ & $\pm \mathrm{DP}$ & $x$ & $\pm \mathrm{DP}$ & $x$ & $\pm \mathrm{DP}$ \\
\hline Nematoda & 932 & 562 & 147 & 123 & 164 & 74,3 & 159 & 97 & 1099 & 144 & 315 & 111 & 573 & 144 & 501 & 366 & 342 & 286 & 519 & 157 & 478,4 & 385,7 \\
\hline Copepoda & 3 & 1,8 & 0.7 & 0.5 & 311 & 219 & 0.7 & 1 & 134 & 49 & 142 & 80,3 & 12.8 & 8,3 & 37,3 & 62,2 & 37,3 & 38,8 & 2 & 2,2 & 68.8 & 120,2 \\
\hline $\begin{array}{l}\text { Larva } \\
\text { Crustacea }\end{array}$ & 1,7 & 3,5 & 0,3 & 0,5 & 106 & 64,4 & 2,3 & 2,6 & 3,5 & 4 & 47 & 37,5 & 11,8 & 7,6 & 47,8 & 90,2 & 9,7 & 7,37 & 0,5 & 0,6 & 23,4 & 47,1 \\
\hline Polychaeta & 5,2 & 2,2 & 1 & 1,4 & 46,3 & 7,5 & 0,3 & 0,5 & 17,8 & 6,8 & 64,5 & 69,1 & 4,3 & 4,6 & 7,3 & 5,8 & 21 & 20,1 & 2 & 1,4 & 14,8 & 20,3 \\
\hline Kinorhyncha & 1,5 & 1,3 & 0,3 & 0,5 & 1,3 & 1,9 & & & 51,8 & 24,4 & 27 & 17,2 & 5,7 & 5,2 & 42,8 & 75,2 & 6,3 & 4,9 & 0,3 & 0,5 & 13,8 & 29,6 \\
\hline Oligochaeta & 0,3 & 0.5 & & & 0,5 & 1 & 1,3 & 1,3 & 2,5 & 3 & 6,5 & 7,4 & 5.5 & 1,7 & 1 & 0,8 & 1 & 1 & 0,5 & 1 & 2 & 3,3 \\
\hline Turbeliaria & 0,3 & 0.5 & & & 11 & 12,9 & & & & & 1.5 & 1,7 & 0.3 & 0,5 & & & 0,3 & 0,6 & & & 1,4 & 5 \\
\hline Acarii & & & & & 1,7 & 1,5 & & & & & 7 & 4,7 & & & & & & & & & 0,9 & 2,6 \\
\hline Tardigrada & & & & & 1,3 & 1,9 & & & & & 2,7 & 3,1 & 0,3 & 0.5 & 1 & 2 & 1.6 & t.5 & & & 0,7 & 1,5 \\
\hline Mysidacea & & & & & & & & & & & & & & & 3,3 & 6,5 & & & & & 0,3 & 2,1 \\
\hline Tanaidacea & & & & & & & & & & & 1,5 & 3 & & & & & & & & & 0,2 & 1 \\
\hline Gastrotricha & & & & & 0,7 & 1,5 & & & & & & & & & & & & & & & 0,1 & 0,5 \\
\hline Isopoda & & & & & & & & & & & & & & & & & 0,3 & 0.6 & & & 0.02 & 0.2 \\
\hline Nemertina & & & & & 0,3 & 0,5 & & & & & & & & & & & & & & & 0,02 & 0,2 \\
\hline Gastropoda & & & & & & & 0.3 & 0,5 & & & & & & & & & & & & & 0,02 & 0,2 \\
\hline
\end{tabular}




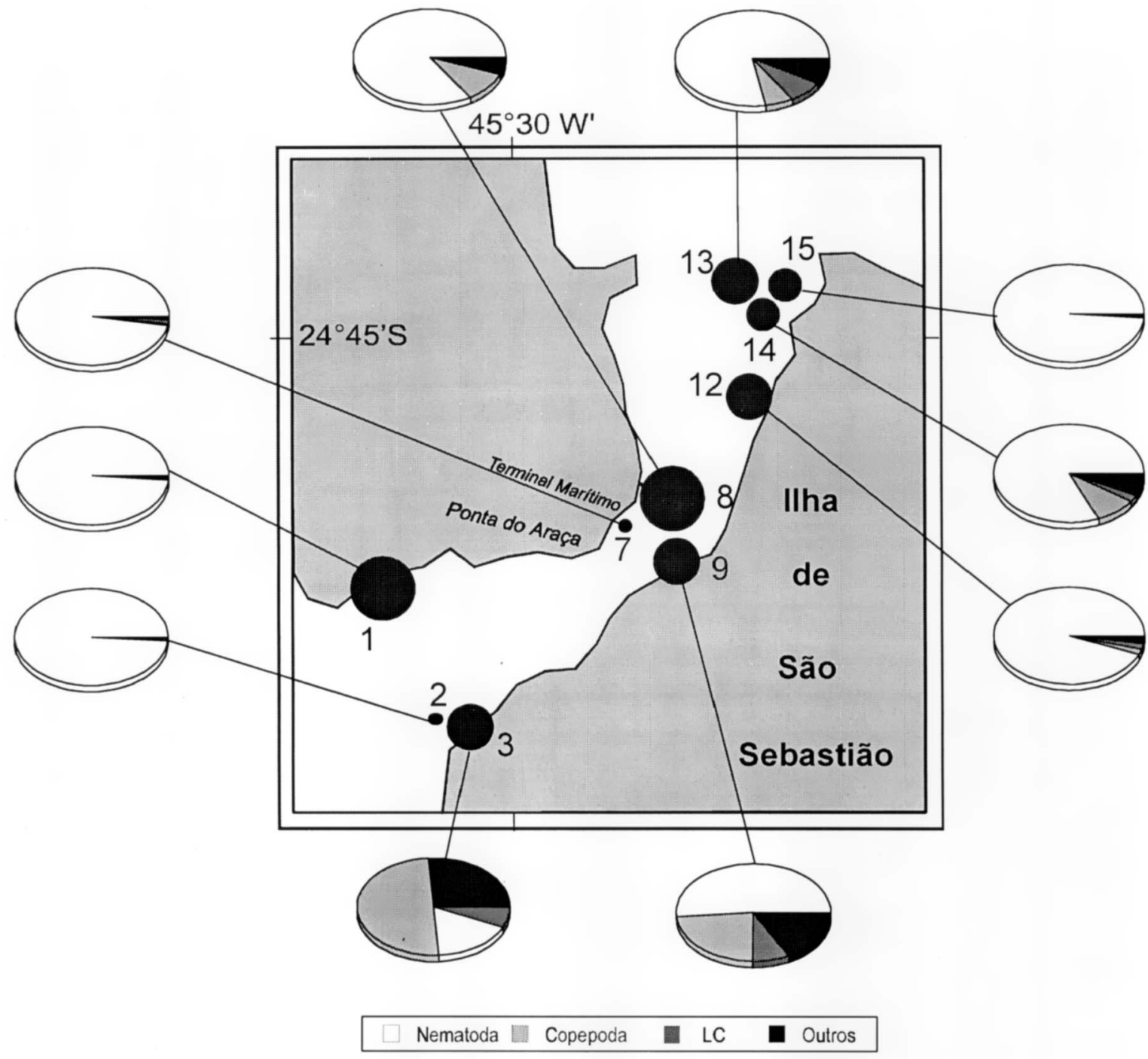

- de 001 a 200 inds. $/ 4,9 \mathrm{~cm}^{2}$

de 401 a 600 inds. $/ 4,9 \mathrm{~cm}^{2}$

de 601 a 800 inds. $/ 4,9 \mathrm{~cm}^{2}$

acima de 801 inds. $/ 4,9 \mathrm{~cm}^{2}$

de 201 a 400 inds. $/ 4,9 \mathrm{~cm}^{2}$

Fig. 2. Representação gráfica da variação espacial da densidade da meiofauna (indivíduos $/ 4,9 \mathrm{~cm}^{2}$ ) e distribuição espacial das freqüências taxonômicas relativas, nas estações de coleta - Verão 1994 (LC - Larva de Crustacea).

estações 2 e 7 (Fig. 2). A biomassa média estimada em termos de peso seco livre de cinzas variou de $31,9 \pm 23,6 \mu \mathrm{g} .4,9 \mathrm{~cm}^{-2}$ (estação 2) a $412,3 \pm 22,5$

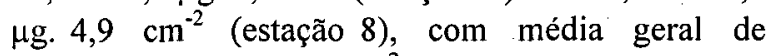
$184,6 \pm 156,9 \mu \mathrm{g} .4,9 \mathrm{~cm}^{-2}$, e sua variação espacial foi semelhante à da densidade.

A análise das diferenças significativas quanto à densidade da meiofauna total e dos Nematoda (Tab. 3) mostrou que as estações 2 e 7 , no setor centro-sul do canal, distinguiram-se das demais pela baixa densidade, bem como as estações 1 e 8, pelas altas densidades, enquanto que o restante mostrou densidades intermediárias. Com relação aos Copepoda, larvas de Crustacea e Polychaeta, principalmente, as estações 2 e 7 também caracterizaram-se por baixas densidades ou ausência desses grupos, enquanto que as estações 3 e 9, do lado insular, com alta porcentagem de areia, apresentaram altas densidades desses grupos, além da estação 8, mais profunda, no centro do canal (Tab. 3). 
Tabela 3. Análise de postos de Kruskal-Wallis das densidades da meiofauna total e dos principais táxons encontrados no Canal de São Sebastião Verão $1994(p<0,05)$.



No inverno, observaram-se 12 grupos taxonômicos (Tab. 4). Os Nematoda aumentaram sua predominância, correspondendo a $90,4 \%$ do total da meiofauna. Os Copepoda reduziram sua porcentagem para 5,3\%, os Polychaeta mantiveram-se em $2,1 \%$ do total e o restante dos grupos constituiu menos de $1 \%$ do total cada. As estações 3, 9, 14 e 15 apresentaram um número maior de grupos taxonômicos encontrados (Fig. 3).

Foi observada uma densidade média geral bem inferior à de verão, $213 \pm 181$ indivíduos por 4,9 $\mathrm{cm}^{2}$, sendo desta $193 \pm 171$ indivíduos do grupo Nematoda (Tab. 4). Verificaram-se as maiores densidades nas estações 3 e 7, e a menor densidade ocorreu na estação 12 (Fig. 3). A biomassa estimada em termos de peso seco livre de cinzas variou de 4,49 $\pm 3,63 \mu \mathrm{g}$. $4,9 \mathrm{~cm}^{-2}$ (estação 12) a $130,19 \pm 30,07 \mu \mathrm{g} .4,9 \mathrm{~cm}^{-2}$ (estação 3 ), com média de $66,6 \pm 48,96 \mu \mathrm{g} .4,9 \mathrm{~cm}^{-2}$, e sua variação espacial também foi semelhante à da densidade.

A análise das diferenças significativas quanto à densidade da meiofauna total e dos Nematoda mostrou que as estações 12,13 e 15, situadas na porção norte, e a 9 , na porção sul do canal, lado insular, distinguiram-se das demais pela baixa densidade (Tab. 5). As maiores densidades foram encontradas nas estações 1 e 3 , na porção sul e na 7, no centro, lado continental. Quanto aos Copepoda, Polychaeta e larvas de Crustacea as estações 7 e 2 , além da estação 12 , caracterizaram-se pelas menores densidades, enquanto que as estações 3 e 9 apresentaram as maiores.

Tabela 4. Média das densidades da meiofauna (no ind. 4,9 cm ${ }^{-2}$ ) nas estações de coleta do Canal de São Sebastião - Inverno 1994 ( $\mathrm{X}=$ média; $\mathrm{DP}=$ desvio padrão).

\begin{tabular}{|c|c|c|c|c|c|c|c|c|c|c|c|c|c|c|c|c|c|c|c|c|c|c|}
\hline \multirow{2}{*}{$\begin{array}{c}\text { ESTAÇÕES } \\
\text { GRUPOS }\end{array}$} & \multicolumn{2}{|c|}{01} & \multicolumn{2}{|c|}{02} & \multicolumn{2}{|c|}{03} & \multicolumn{2}{|c|}{07} & \multicolumn{2}{|c|}{08} & \multicolumn{2}{|c|}{09} & \multicolumn{2}{|c|}{12} & \multicolumn{2}{|c|}{13} & \multicolumn{2}{|c|}{14} & \multicolumn{2}{|c|}{15} & \multicolumn{2}{|c|}{ TOTAL } \\
\hline & $x$ & $\pm \mathrm{DP}$ & $x$ & $\pm \mathrm{DP}$ & $x$ & $\pm \mathrm{DP}$ & $x$ & $\pm \mathrm{DP}$ & $x$ & $\pm \mathrm{DP}$ & $x$ & $\pm \mathrm{DP}$ & $x$ & $\pm \mathrm{DP}$ & $x$ & $\pm \mathrm{DP}$ & $x$ & $\pm \mathrm{DP}$ & $x$ & $\pm \mathrm{DP}$ & $x$ & $\pm \mathrm{DP}$ \\
\hline Nematoda & 308 & 101 & 161 & 129 & 382 & 83,4 & 408 & 317 & 237 & 105 & 67 & 35,4 & 12,5 & 9,6 & 88,3 & 17 & 187 & 80 & 80,5 & 34,3 & 193 & 171,4 \\
\hline Copepoda & 1,5 & 1,7 & & & 59,3 & 23,6 & & & 4,5 & 4,2 & 26,8 & 15,8 & & & 1 & 1,4 & 8,7 & 3,9 & 11,8 & 14,4 & 11,3 & 20,2 \\
\hline Polychaeta & 1,5 & 1,3 & 0,7 & 0,9 & 10 & 2 & 1,7 & 0,5 & 6 & 4,7 & 16,5 & 10,5 & & & 1,5 & 1,9 & 5,5 & 4,1 & 2.3 & 1.7 & 4,5 & 6.2 \\
\hline $\begin{array}{l}\text { Larva } \\
\text { Crustacea }\end{array}$ & & & & & 8 & 6.1 & & & & & 6,3 & 7,6 & 0,3 & 0,5 & 0,3 & 0,5 & 2 & 2,7 & 1,3 & 1,5 & 1,8 & 4 \\
\hline Oligochaeta & 0,3 & 0,5 & 0,7 & 0,5 & 1,3 & 1,3 & 0,7 & 1 & 3,3 & 1,9 & & & & & 1,7 & 0,5 & 2 & 1,4 & 0,3 & 0.5 & 1 & 1.3 \\
\hline Turbellaria & 0,3 & 0,5 & & & 2,5 & 2,4 & & & & & 0.5 & 0,6 & & & 0,3 & 0,5 & 1,3 & 1,3 & 0,3 & 0.5 & 0.5 & 1,1 \\
\hline Kinorhyncha & & & & & 2,5 & 2,4 & & & 0,5 & 0,6 & 0,5 & 1 & & & & & 0,3 & 0,5 & 0,7 & 1 & 0,4 & 1,1 \\
\hline Bivalvia & & & & & 1 & 2 & & & 0,7 & 1 & 0,5 & 1 & & & & & 0,3 & 0,5 & & & 0,3 & 0.7 \\
\hline Ostracoda & & & & & 2,3 & 2.2 & & & & & & & & & & & & & & & 0,3 & 0.9 \\
\hline Tardigrada & & & & & & & & & & & 1 & 2 & & & & & 0,7 & 1,5 & & & 0,2 & 0,8 \\
\hline Gastrotricha & 0,3 & 0,5 & & & 0.3 & 1 & & & & & & & & & & & & & & & 0.1 & 0.2 \\
\hline Acarii & & & & & & & & & & & 0,3 & 0,5 & & & & & & & & & 0,1 & 0,12 \\
\hline TOTAL & 311 & 102 & 162 & 130 & 469 & 106 & 410 & 318 & 252 & 112 & 120 & 55,1 & 12,8 & 9,7 & 93 & 17,4 & 208 & 89 & 97 & 42,1 & 213,4 & 180,8 \\
\hline
\end{tabular}




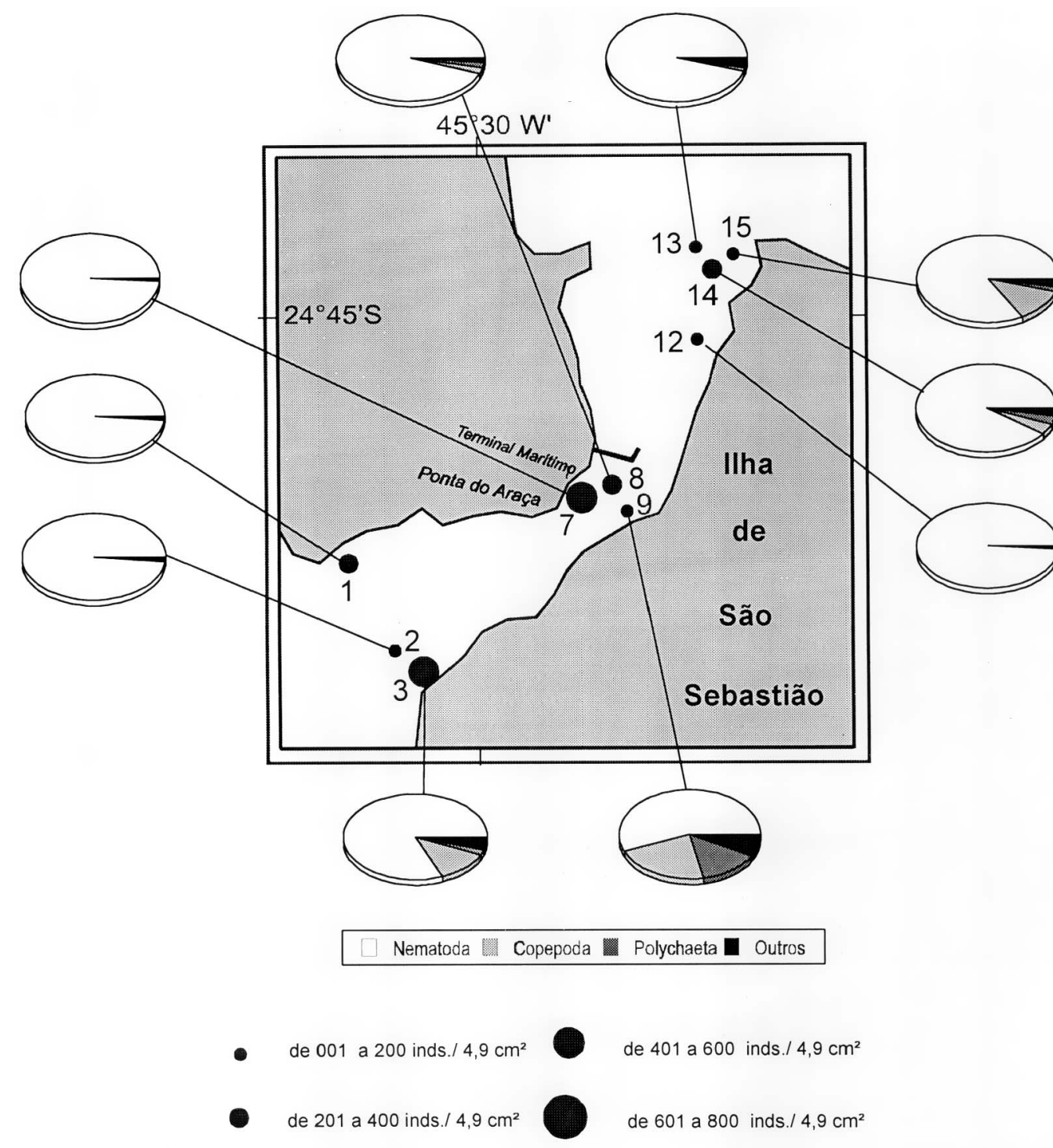

Fig. 3. Representação gráfica da variação espacial da densidade da meiofauna (indivíduos $/ 4,9 \mathrm{~cm}^{2}$ ) e distribuição espacial das freqüências taxonômicas relativas, nas estações de coleta - Inverno 1994.

A análise de correspondência canônica (ACC) indicou que os principais fatores responsáveis pela distribuição dos grupos da meiofauna e das estações foram os relacionados ao sedimento, principalmente o diâmetro médio dos grãos, o teor de carbonatos e o grau de seleção, além da temperatura e a profundidade (Fig. 4). $\mathrm{O}$ eixo 1 explicou $81,1 \%$ da ordenação e distribuiu as estações em um gradiente de areia grossa a silte. Houve um grupo constituído pelas estações 1 e 2 , com sedimentos mais finos, maior teor de carbono orgânico e com predominância dos Nematoda, situadas principalmente do lado continental. Outro grupo, com as estações 3 e 9, ao longo do lado insular, foi caracterizado pela maior quantidade de areia e carbonatos no sedimento, com maior representação de Copepoda e larvas de Crustacea. Houve outro conjunto de estações que apresentou características variáveis, em relação à meiofauna e ao sedimento, dependendo da época do ano. Este grupo esteve situado principalmente na porção norte do canal, como as estações 12,13 e 14, assim como na parte central do canal (estação 8). O grupo das estações 12 a 14 do período de verão distinguiu-se do inverno em função do melhor grau de selecionamento do sedimento, da temperatura da água mais alta e da maior representatividade das larvas de Crustacea. No inverno, de um modo geral, houve maior predominância de areia e/ou maior teor de carbonatos nessas estações (eixo 2). 
Tabela 5. Análise de postos de Kruskal-Wallis das densidades da meiofauna total e dos principais táxons encontrados no Canal de São Sebastião - Inverno $1994(\mathrm{p}<0,05)$.

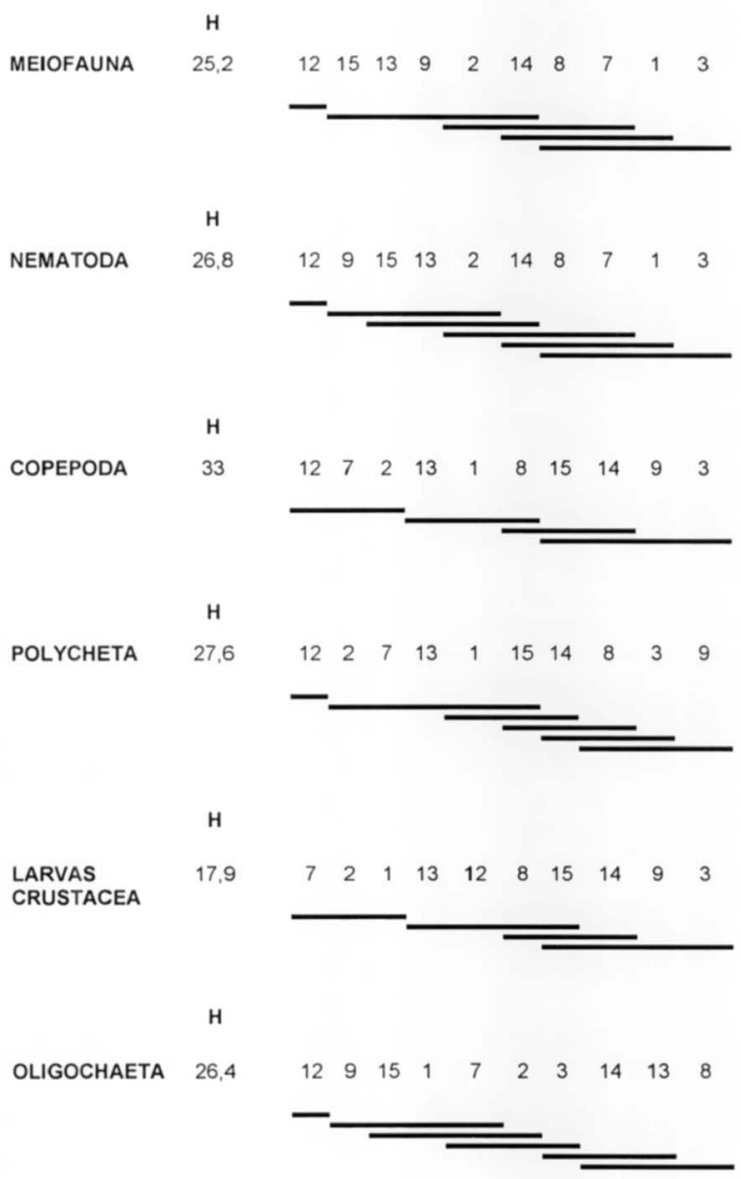

\section{Discussão}

Segundo Furtado (1995), a sedimentação atual da área encontra-se intimamente ligada à circulação geral, à topografia irregular do fundo do canal e a sua geometria. A porção continental do canal é tipicamente de deposição, enquanto o lado insular apresenta tendências à erosão. No eixo do canal, ocorrem misturas de areia e lamas ocorrendo, na porção norte, a deposição de sedimentos mais grossos mal selecionados, resultantes da aceleração do fluxo na área central e uma resultante para NE no transporte sedimentar. No sul, junto ao continente, os sedimentos são compostos por areias finas, com bom grau de seleção, devido ao retrabalhamento dos sedimentos pela ação das ondas. Duas estações do lado insular localizam-se próximo a costões cristalinos, que atuam como fontes para areias, sendo o aporte de argilas para o local incipiente.
Canal de São Sebastião

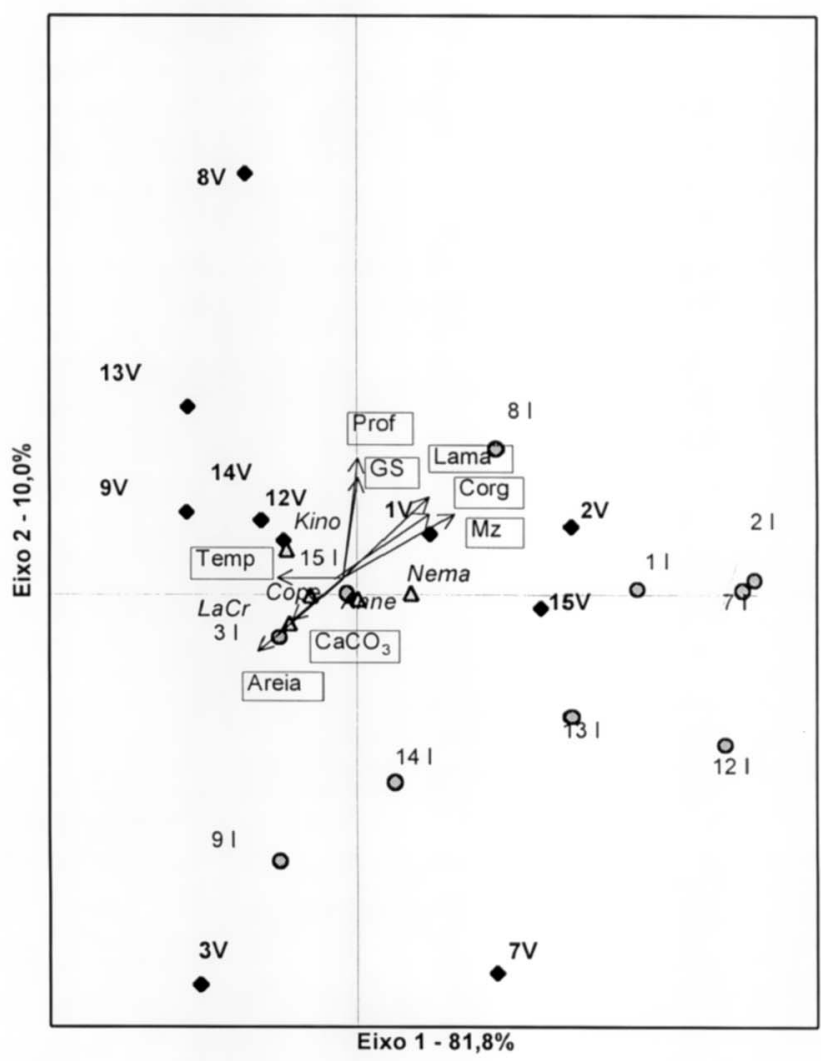

Fig. 4. Diagrama da ordenação ACC da meiofauna do Canal de São Sebastião: biplot dos grupos taxonômicos $(\Delta)$ e das estações com as variáveis ambientais $(\rightarrow)$. Estações de fevereiro/94 $(\bullet)$ e de agosto/94 $(\bullet)$. (Prof. - profundidade; GS - grau de seleção; Corg carbono orgânico; $\mathrm{Mz}$ - diâmetro médio do grão; Temp - temperatura; Kino - Kinothyncha; Nema Nematoma; Anne - Annelida; LaCr - Larva de Crustacea; Cope - Copepoda).

Estes aspectos de circulação explicam as alterações no sedimento observadas entre o verão e o inverno, principalmente na porção norte. Esta heterogeneidade na distribuição dos sedimentos refletiu-se na distribuição da meiofauna bentônica.

O principal fator determinante da composição e distribuição da meiofauna é o tipo de sedimento e suas características. Vários autores, entre eles McLachlan et al. (1977) e Coull (1988), ressaltam que sedimentos com grãos mais grossos apresentam maior espaço entre os grãos, o que promove uma maior variedade de nichos que passam a ser ocupados por outros indivíduos além dos Nematoda, como os Copepoda e Polychaeta. Os Nematoda, ao contrário dos Copepoda, são alongados e melhor adaptados aos finos interstícios, podendo ser encontrados $\mathrm{em}$ sedimentos mais finos; também há diversas espécies do grupo que são tolerantes ou adaptadas às condições anóxicas que existem logo abaixo dos 
primeiros centímetros de sedimentos lamosos (Jensen, 1987). A dominância dos Nematoda na maioria das estações é uma característica da meiofauna, já que este táxon geralmente domina a meiofauna perfazendo mais de $50 \%$ do número total de indivíduos (Coull, 1988), principalmente em sedimentos finos do sublitoral.

$\mathrm{O}$ conjunto desses fatores refletiu-se nas características da meiofauna do Canal de São Sebastião, que variou espacialmente na abundância total, entre o verão e o inverno, como também em relação à densidade dos diferentes táxons, nos dois períodos e entre as estações. Tanto no verão como no inverno, as estaçães onde as densidades de grupos como Copepoda, principalmente, e Polychaeta, Kinorhyncha e larva de Crustacea foram mais significativas, foram as dominadas pelas frações arenosas. Já as estações cujo sedimento foi dominado pelas frações sílticas, as densidades de Nematoda foram altas e os outros táxons não foram encontrados ou suas densidades eram muito baixas. A exceção a este padrão são as estações 2,7 e 8 .

As estações 2 e 7 , durante o verão, apesar da maior porcentagem da fração síltica, apresentaram as mais baixas densidades, o que pode estar relacionado com os níveis de influência antrópica na área, já que ambas as estações são as que possuem os teores de carbono orgânico mais altos do canal (Barcellos \& Furtado, 1999). Na região da estação 7 há a descarga de um emissário submarino de esgoto, cujo despejo é maior no verão, época de intensa atividade turística na região, bem como há influência do DTCS (Dutos e Terminais do Centro Sul) e do porto de São Sebastião. Weber et al. (1998) encontraram no sedimento da região contribuição de hidrocarbonetos de petróleo, o mesmo acontecendo na região da estação 2. Esta, por sua vez, pode estar sob efeito de lavagem ilegal de tanques de navios petroleiros, nesta região da plataforma interna de São Sebastião (Zanardi, 1996).

A estação 8, a mais profunda, no verão apresentou temperatura da água de fundo mais baixa e a densidade total foi a maior dentre todas as estações do canal, com uma alta densidade de Copepoda e a maior densidade de Kinorhyncha, embora 0 sedimento fosse constituído de silte médio com um alto valor de carbono orgânico. Estes fatores desfavorecem a sobrevivência de grupos como os Copepoda, como citado anteriormente. No entanto, a porcentagem de carbonatos biodetríticos foi a terceira maior da região do canal, abaixo somente das estações 3 e 9 . Supõe-se que o carbonato, que nesta estação está refletindo a maior contribuição de resíduos de cascalho no sedimento, favoreça a porosidade no sedimento muito fino, aumentando os interstícios entre os grãos, o que possibilitaria a sobrevivência de outros grupos além dos Nematoda. Outro fator possível seria a presença de resquícios da ACAS nessa estação, durante o verão, quando os teores de nitrogênio do sedimento foram os mais altos (Barcellos \& Furtado, 1999).

Comparando o verão com o inverno, houve uma redução da densidade da meiofauna de cerca de três vezes. No canal, a perturbação física do sedimento é maior durante o inverno quando a passagem de sistemas frontais é mais freqüente. Estes sistemas apresentam ventos com intensidade suficiente para causar ondas que revolvem e redistribuem o sedimento (Tessler, 1988), especialmente em profundidades inferiores a $25 \mathrm{~m}$ (Furtado, com. pessoal*). Distúrbios físicos no sedimento em função do hidrodinamismo podem causar a ressuspensão para a coluna d'água e a remoção da meiofauna que tem densidade próxima à da água do mar (Palmer, 1984; Thistle, 1995). Este tipo de perturbação foi constatado em relação à macrofauna da plataforma interna de Ubatuba (PiresVanin, 1992 e 1993; Paiva, 1993, 1994). Na Enseada do Flamengo, uma área mais protegida e costeira em Ubatuba, Silva (1996) também encontrou baixas densidades da meiofauna durante o inverno. GuidiGuilvard \& Buscail (1995), ao estudarem a meiofauna em um ponto na saída do Rio Tet, a noroeste do mar Mediterrâneo, observaram que o fator principal que afetou a densidade da meiofauna foram as fortes correntes de fundo geradas pelos fortes ventos na superfície do mar. Como ressaltado pelas autoras, a meiofauna presente nas camadas superficiais do sedimento facilmente passa para a coluna d'água, podendo até ser transportada para outros locais (Palmer, 1988).

A entrada da ACAS na plataforma interna do litoral norte de São Paulo, durante a primavera-verão, promove um aumento da produtividade primária fitoplanctônica e, conseqüentemente, uma maior quantidade de recursos alimentares pode ser canalizada para o bentos (Pires-Vanin, 1993). Os resultados da densidade média da meiofauna do Canal de São Sebastião, no verão $\left(1210,8\right.$ ind. $\left.10 \mathrm{~cm}^{-2}\right)$, foram maiores do que os obtidos na plataforma interna ao largo de Ubatuba, SP (723 ind. $\left.10 \mathrm{~cm}^{-2}\right)$, no verão de 1989 (Corbisier, 1993) e maiores que de outras regiões de plataforma como citado em Corbisier (1993), refletindo possivelmente uma contribuição costeira mais expressiva na área, bem como, uma maior variabilidade sedimentar.

Em relação à biomassa, os valores obtidos em São Sebastião, no verão, foram menores do que os de Ubatuba (Corbisier, 1993), indicando que os organismos da meiofauna do canal, apesar de numerosos, foram menores que os daquela região.

Os resultados de duas amostragens anuais ainda não podem ser considerados como o padrão de comportamento temporal da meiofauna na região do canal. As coletas realizadas durante ou logo após a

(*) Furtado, V. V. 2000. Universidade de São Paulo, Instituto Oceanográfico. 
passagem de sistemas frontais devem refletir o efeito de revolvimento do fundo e, vários autores, entre eles Coull (1988) e Guidi-Guilvard \& Buscail (1995), ressaltam que os ciclos da meiofauna são altamente variáveis. Desse modo, variações temporais devem ser analisadas com maior detalhamento para a busca de padrões, uma vez que estes organismos são capazes de recolonizar um sedimento perturbado em um prazo de dias a semanas (Coull, 1988).

\section{Agradecimentos}

Este projeto recebeu apoio financeiro da FAPESP (proc. no 92/3449-0, Projeto OPISS). Agradecemos ao $\mathrm{CNPq}$ pelas Bolsas de Mestrado, Aperfeiçoamento e Auxílio Integrado à Pesquisa (proc. 530388/93-6) concedidas a este projeto; à Bióloga Émile F. Silveira, pelo auxílio na triagem das amostras; e à equipe científica dos Cruzeiros Oceanográficos do Projeto OPISS pela coleta das amostras.

\section{Referências bibliográficas}

Aller, J. Y. \& Aller, R. C. 1986. General characteristics of benthic faunas on the Amazon inner continental shelf with comparison to the shelf off the Changjiang River, East China Sea. Continent. Shelf Res., 6(1-2):291-310.

Alongi, D. M. 1989. Benthic processes across mixed terrigenous-carbonate sedimentary facies on the central Great Barrier Reef continental shelf. Continent. Shelf Res., 9(7):629-663.

Alongi, D. M. 1990. The ecology of tropical softbottom benthic ecosystems. Oceanogr. mar. Biol. a. Rev., 28:381-496.

Barcellos, R. L. \& Furtado, V. V. 1999. Processo sedimentar atual e a distribuição de carbono e nitrogênio orgânicos no Canal de São Sebastião (SP) e plataforma continental interna adjacente. Rev. bras. oceanogr., 47(2):207-221.

Bartlett, P. D. \& Henning, H. F-K. O. 1987. Pollution studies in False Bay, South Africa: chemical versus meiofaunal indicators. In: Cappuzo, J. M. \& Kester, D. R. eds. Oceanic processes in marine pollution. v. 1 Biological processes and wastes in the ocean. Malabar, Robert E. Krieger Publishing Company. p. 231-240.
Bodin, P.; Boucher, D. \& Le Guellec, C. 1989. Estimation des biomasses du microphytho- et du méiobenthos en Baie de Saint-Brieuc. Relat. Contrat I.F.R.E.M.E.R./U.B.O $\mathrm{n}^{\circ}$ 88.2.43.0426. $35 \mathrm{p}$.

Castro Filho, B. M. de; Miranda, L. B. de \& Miyao, S. Y. 1987. Condições hidrográficas na plataforma continental ao largo de Ubatuba: variações sazonais e em média escala. Bolm Inst. oceanogr., S Paulo, 35(2):135-151.

Corbisier, T. N. 1993. Meiofauna da plataforma continental interna do litoral norte de São Paulo verão/89. Publção esp. Inst. oceanogr., S Paulo, (10):123-135.

Coull, B. C. 1988. Ecology of the marine meiofauna. In: Higgins, R. P. \& Thiel, H. eds. Introduction to the study of meiofauna. Washington, Smithsonian Institution Press. p. 18-38.

Coull, B. C. \& Chandler, G. T. 1992. Pollution and meiofauna: field, laboratory and mesocosm studies. Oceanogr. mar. Biol. a. Rev. 30: 191-271.

Furtado, V. V. 1995. Sedimentação quaternária no Canal de São Sebastião. Publção esp. Inst. oceanogr., S Paulo, (11):27-35.

Furtado, V. V.; Bonetti, J.; Rodrigues, M. \& Barcellos, R. L. 1998. Aspectos da sedimentação no Canal de São Sebastião. Relat. téc. inst. oceanogr., (43):15-31.

Gaudette, H. E.; Flight, W. R.; Toner, L. \& Folger, D. W. 1974. An inexpensive titration method for the determination of organic carbon in recent sediments. J. sedim. Petrology, 44(1):249-253.

Guidi-Guilvard, L. D. \& Buscail, R. 1995: Seasonal survey of metazoan meiofauna and surface sediment organics in a non-tidal turbulent sublittoral prodelta (northwestern Mediterranean). Continent. Shelf Res., 15(6): 633-653.

Hanson, R. B.; Tenore, K. R.; Bishop, S.; Chamberlain, C.; Pamatmat, M. M. \& Tietjen, J. 1981. Benthic enrichment in the Georgia Bight related to Gulf Stream intrusions and estuarine outwelling. J. Mar. Res., 39(3):417-441.

Jensen, P. 1987. Differences in microhabitat, abundance, biomass and body size between oxybiotic and thiobiotic free-living marine nematodes. Oecologia, 71:564-567. 
Jongman, R. H.; Ter Braak, C. J. F. \& Van Tongeren, O. F. R. 1987. Data analysis in community and landscape ecology. Wageningen, PUDOC. 299 p.

Mahiques, M. M.; Mishima, Y. \& Rodrigues, M. 1999. Characteristics of the sedimentary organic matter on the inner and middle continental shelf between Guanabara Bay and São Francisco do sul, southeastern Brazilian margin. Continent. Shelf Res., 19:775-798.

McLachlan, A.; Winter, P. E. D. \& Botha, A. L. 1977. Vertical and horizontal distribution of sub-littoral meiofauna in Algoa Bay, South Africa. Mar. Biol., 40(4):355-364.

Paiva, P. C. de. 1993. Anelídeos Polychaeta da plataforma continental norte do Estado de São Paulo: I - Padrões de densidade e diversidade específica. Bolm Inst. oceanogr., S Paulo, 41(12):69-80.

Paiva, P. C. de. 1994. Trophic structure of a shelf polychaete taxocenosis in southern Brazil. Cah. Biol. mar. 35(1):39-55.

Palmer, M. A. 1984. Invertebrate drift: behavioral experiments with intertidal meiobenthos. Mar. Behav. Physiol., 10(3):235-253.

Palmer, M. A. 1988. Dispersal of marine meiofauna: a review and conceptual model explaining passive transport and active emergence with implications for recruitment. Mar. Ecol. Prog. Ser., 48(1):8191.

Pfannkuche, O. \& Thiel, H. 1988. Sample processing. In: Higgins, R. P. \& Thiel, H. eds. Introduction to the study of meiofauna. Washington, Smithsonian Institution Press. p. 134-145.

Pires-Vanin, A. M. S. 1992. Structure and dynamics of benthic megafauna on the continental shelf offshore of Ubatuba, southeastern Brazil. Mar. Ecol. Prog. Ser., 86(1):63-76.

Pires-Vanin, A. M. S. 1993. A macrofauna bêntica da plataforma continental ao largo de Ubatuba, São Paulo, Brasil. Publção. esp. Inst. Oceanogr., S. Paulo, (10):137-158.

Rudnick, D. T.; Elmgren, R. \& Frithsen, J. B. 1985. Meiofaunal prominence and benthic seasonality in a coastal marine ecosystem. Oecologia, 67(2):157168 .

Silva, J. da. 1996. Meiofauna da Enseada do Flamengo, Ubatuba-SP. M.Sc. thesis. Universidade de São Paulo, Instituto Oceanográfico. $122 \mathrm{p}$.
Suguio, K. 1973. Introdução à sedimentologia. São Paulo. Edgard Blucher/EDUSP. 317 p.

Tenore, K. R.; Chamberlain, C. F.; Dunstan, W. M.; Hanson, R. B.; Sherr, B \& Tietjen, J. H. 1978. Possible effects of Gulf Stream intrusions and coastal runoff on the benthos of the continental shelf of Georgia Bight. In: Wiley, M. L. ed. Estuarine interactions. New York, Academic Press. p. 577-598.

ter Braak, C. J. F. 1988. CANOCO - a FORTRAN program for canonical communiy ordination by partial detrended canonical correspondence analysis, principal components analysis and redundancy analysis (version 2.1). Wageningen, Agricultural Mathematics Group. 95 p.

ter Braak, C. J. F. 1990. Update Notes: CANOCO Version 3.10. Wageningen, Agricultural Mathematics Group. 35 p.

Tessler, M. G. 1988. Dinâmica sedimentar quaternária no litoral sul paulista. PhD. thesis. Universidade de São Paulo, Instituto de Geociências. 277 p.

Thistle, D.; Weatherly, G. L. \& Ertman, S. C. 1995. Shelf harpacticoid copepods do not escape into the seabed during winter storms. J. mar. Res., $53(5): 847-863$.

Weber, R. R.; Zanardi, E. \& Bícego, M. C. 1998 Distribuição e ocorrência dos hidrocarbonetos biogênicos e de petróleo, na água do mar superficial e nos sedimentos de superfície da região da plataforma interna de São Sebastião, SP, Brasil. Relat. téc. inst. oceanogr., (43):1-14.

Widbom, B. 1984. Determination of average individual meiofauna dry weights and ash-free dry weights in different sieve fractions of marine meiofauna. Mar. Biol., 84(1):101-108.

Zanardi, E. 1996. Hidrocarbonetos no canal de São Sebastião e na plataforma interna adjacente influência do derrame de maio de 1994. M.Sc. thesis. Universidade de São Paulo, Instituto Oceanográfico. $112 \mathrm{p}$.

Zar, J. H. 1996. Biostatistical analysis. $3^{\text {rd }}$. ed. Englewood Cliffs, Prentice-Hall. 661 p.

(Manuscrito recebido 23 maio 2001; revisado 08 agosto 2001; aceito 08 março 2002) 